\title{
Structure Design and Simulation of A Bionic Continuous Hopping Robot

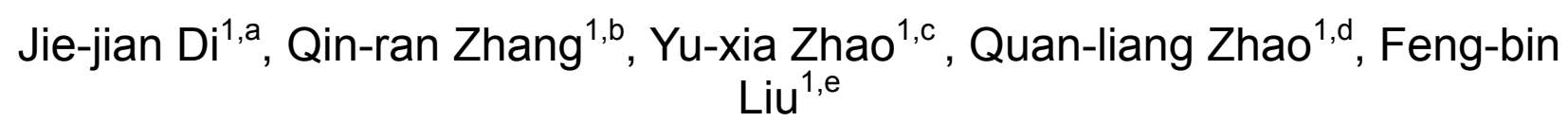

${ }^{1}$ School of Mechanical and Materials Engineering, North China University of Technology,

\author{
Beijing 100041, China

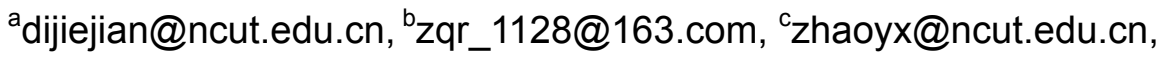 \\ dzql-01@163.com, effliu@ncut.edu.cn
}

Keywords: Bionic, hopping robot, kinematics simulation

\begin{abstract}
The paper presented a new simple and novel design of a bionic frog-hopping robot to achieve continuous hopping. The flexible jumping leg is made by spring leaf of $\mathrm{C}$-shape, which is beneficial to increase the storage of elastic energy and improve service life. The synchronize compression process of two jumping legs can be achieved by the rotating cam. The cam mechanism not only improves the reliability of the compression process, but also simplifies the design of the control system. The kinematics simulation of bionic frog hopping robot is performed based on ADAMS virtual prototype technology. The results show that the robot can complete three consecutive jumps within 1.3 seconds and the average jumping height is about 0.14 meter, and the total forward distance is about 0.23 meter. The jumping trajectory of the bionic frog-hopping robot is consistent with the desired track. The structure is proved reasonable and the continuous hopping seems to be stable.
\end{abstract}

\section{Introduction}

With the development of science and technology, traditional robot technology can not meet the needs of tasks under varying environmental conditions, more and more robots which can service for special environmental conditions emerge at a historic moment. The bionic robot with high environmental adaptability and reliability gradually become the mainstream research. Bionic robots shows excellent over obstacle ability in terrain surveying, rescue and military reconnaissance activities, and it also be able to enter the dangerous places where humans can not to set foot in [1]. Especially, when the ground environment is soft and damp, the scope of activities of traditional wheeled robots will be greatly restricted.Therefore, the development of bionic hopping robot will be the key to solve those problems.

In recent years, a lot of terrific bionic hopping robot spring up, such as the German company Festo developed the kangaroo hopping robot, not only shaped like a kangaroo, but also inherited the Kangaroo outstanding leaping ability [2]. In order to enable the kangaroo robot which is about one meter high and seven kilograms weight to hop easily and continuously like a real kangaroo, R \& D personnel mounted a fixed spring means in the robot's legs. This structure helps the robot to imitate real kangaroos foot jumping motion, and also can play a buffer role during hopping. In addition, the bionic dog hopping robot Kenken developed by The University of Tokyo and the bionic cricket hopping robot developed by Case Western Reserve University are also excellent bionic hopping robot $[3,4,5]$. The size of the bionic cricket hopping robot is only $5 \mathrm{~cm}$, researchers used polymer materials to imitate the tendons of cricket legs to achieve tension and contraction. As an developing country, technology-driven productivity, China has begun to develop bionic hopping robots. After studying the principles of joints kinematics during the frog's hopping movement, Wang Meng, Harbin Institute of Technology, discovered the frog's hopping law. On this basis, he developed a bionic frog hopping robots using the motor as power source[6]. The bionic frog robot can achieve intermittent hopping by assembling the spring means and five-bar mechanism in legs. However, the 
robot's excellent hopping ability is often directly proportional to the complexity of assembly structures and control systems. So the simple structure and stable hopping ability are important.

As we all know, due to the force of gravity, organism often need to withstand twice the force of its own weight during hopping. Particularly at the previous jumping moment, organisms are in the overweight status, which led to a much greater force than its own gravity happened between the legs and the ground, and this force most to be borne by the organism's leg tendon[7,8]. Based on above studies, a new type of jumping leg is designed for bionic frog-hopping robot. Compared with the traditional jumping leg, the new jumping leg has better flexibility and a bigger storage capacity of elastic potential energy. Another innovation of the design is that the two jumping legs can complete the processing of compression or release in synchronously controlled by the one cam.

\section{The Structure Design of Robot.}

In this section, the structure design of bionic frog-hopping robot will be mainly introduced in two parts, the design of the cam mechanism and jumping leg. The cam mechanism ensures the synchronization of the two jumping legs. And the jumping leg as another important part of a bionic jumping robot, it will bear the action of jumping, storage, and buffering.

The Design of Cam Mechanism. During the ready moment of jumping, the two jumping legs have to be guaranteed to be released synchronously. Otherwise, the robot will rollover under the asymmetric elasticity which is caused by the non synchronously release of the spring. In the cam mechanism, the cam will be driven for rotating by a gear set which is driven by a little motor. Then the cam drives the body to be lower, while the two jumping legs will complete the compression process. So it guarantees that the two jumping legs will achieve the release or compressing under the controlling of one cam.

Based on the above, the cam mechanics is designed. Specific method is that the motor as the original motivation, after the gear-set decelerating, the cam fixed on the end of the gear-set output shaft (gear-set installed in the body, they can be considered as a whole. Jumping legs are respectively mounted on both sides of the body, shown in Fig. 1). After the cam rotates while, it will contact the bearing which is fixed on the bottom of the body. (The bearing can rotate but the height which is relative to the ground will not change). The bearing is driven by the force of the cam to rotate in clockwise, relatively, the cam will also receive the resistance of the bearing. So the body will move down with the cam, completing the compression of spring, shown in Fig. 2.

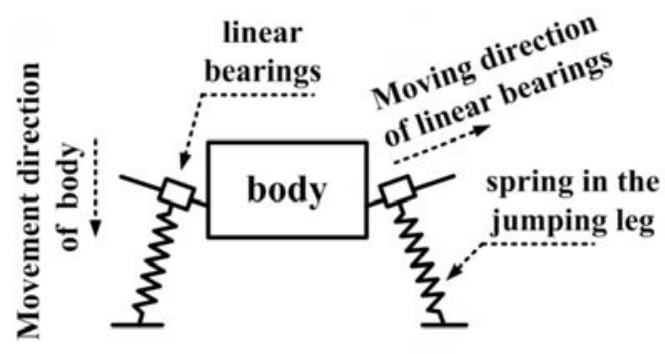

Fig. 1 The robot structure

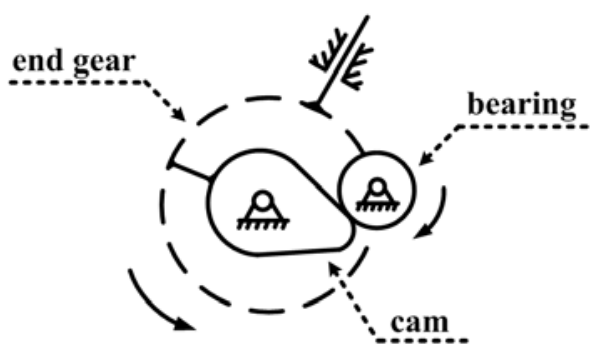

Fig. 2 Working principle of cam

When the cam rotates to a certain angle, the bearing could not provide the resistance force any more. Then the spring will restore to the balance position, and the thigh will impact barrier plate to realize jumping. It is worth mentioning that the motor will make the cam finishing a fully compressed during the vacating state by selecting the appropriate speed. A fully compression cycle is defined from the moment that the cam lose the contacting with the bearing until the next moment the cam almost lose the contacting but still contacting with the bearing. If the cam can complete a fully compression before the landing moment, the spring will be kept with the maximum elastic potential energy once again. So this landing moment will become the next jumping moment. Therefore, the robot can jumping continuously.

The Design of Jumping Leg. Jumping leg as the key part of storage and release of the elastic potential energy, it is required to have a high energy storage capacity. In addition, the jumping leg will be the first part of the robot to contact with the ground when the robot is landing. As an 
important buffer role, it also bears a big impact forces at the landing moment.

The traditional jumping leg is composed of thigh, knee, crus, ankle and foot in five parts, torsion spring is fixed in each joint. This assembly structure is complicated with a large friction. The new type of jumping leg model is simplified into one part with a single C-shape sheet ( supporting foot ) from three parts ( crus, ankle and foot ). This new type jumping leg not only avoids the friction between the parts assembling effectively, but also improves the storage capacity of elastic potential energy. With a better flexibility, C-shape spring sheet will help the bionic frog hopping robot completing jumping successfully. A pressure spring device for storing elastic potential energy is arranged between the thigh and the supporting foot. The jumping leg of the traditional and new type is shown in Fig. 3 and Fig. 4 respectively.

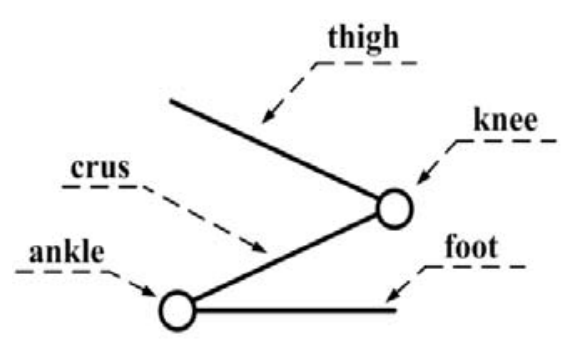

Fig. 3 The traditional jumping leg

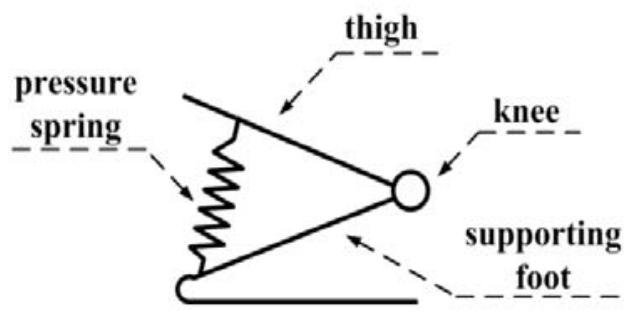

Fig. 4 The new type jumping leg

\section{Kinematic Simulation of Hopping}

Establishment of Simulation Model. First of all, the 3D model of the bionic frog hopping robot is built with Solidworks software, the 3D model is shown in Fig. 5. For convenience, the 3D model is simplified (shown in Fig. 6).

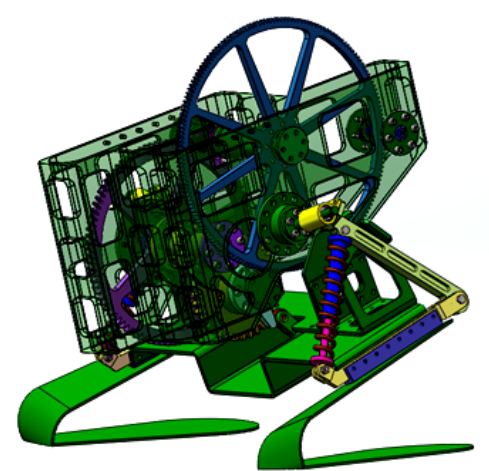

Fig.5 The 3D model in Solidworks

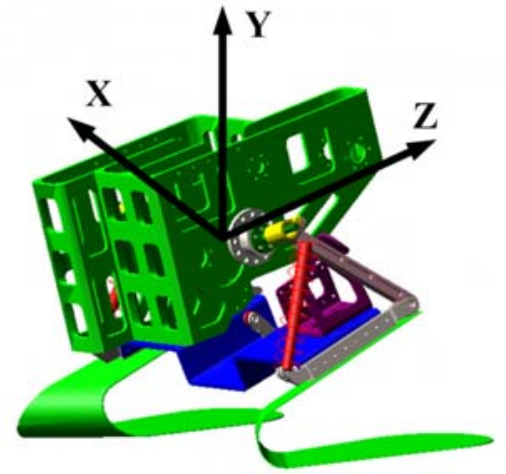

Fig.6 The simplified 3D model in Adams

As can be seen from the Figure 6, the negative direction of the Y axis is the gravity direction, the direction of the $\mathrm{Z}$ axis is the forward jumping direction, and the jumping movement happens in the $\mathrm{YZ}$ plane. The gravity acceleration is $9.8 \mathrm{~m} / \mathrm{s}^{2}$, the friction coefficient is 0.30 , the rotation speed of cam is $1200 \%$ s, the elastic coefficient of spring is $9669 \mathrm{~N} / \mathrm{m}$. In the simulation process, except for the spring, the other parts can be regarded as rigid body, the assembly error and the friction between the parts are negligible.

Simulation Results. The jumping process's simulation of the bionic frog hopping robot is shown in Fig. 7. The picture I shows that the robot is in a jumping preparation moment, while the spring is in a natural states, the cam is rotating but still not contacting with bearing; The picture II shows that the body has already moved down because the cam is contacting with the bearing, the spring is compressed, and robot is ready for jumping; The picture III shows that the spring has released, the robot is in vacating moment, and the cam till rotate for completing the fully compression in the air before the landing moment; The last picture IV shows that the robot is landing, and it also can be seen from the picture IV that the body is in the same position compared to the picture II, so it is obvious that the cam already complete a fully compressed in vacating moment (in picture III). At the same time, the robot is ready for next jumping. The bionic frog-hopping robot has completed 3 consecutive and smooth jumping in 1.3 seconds. 


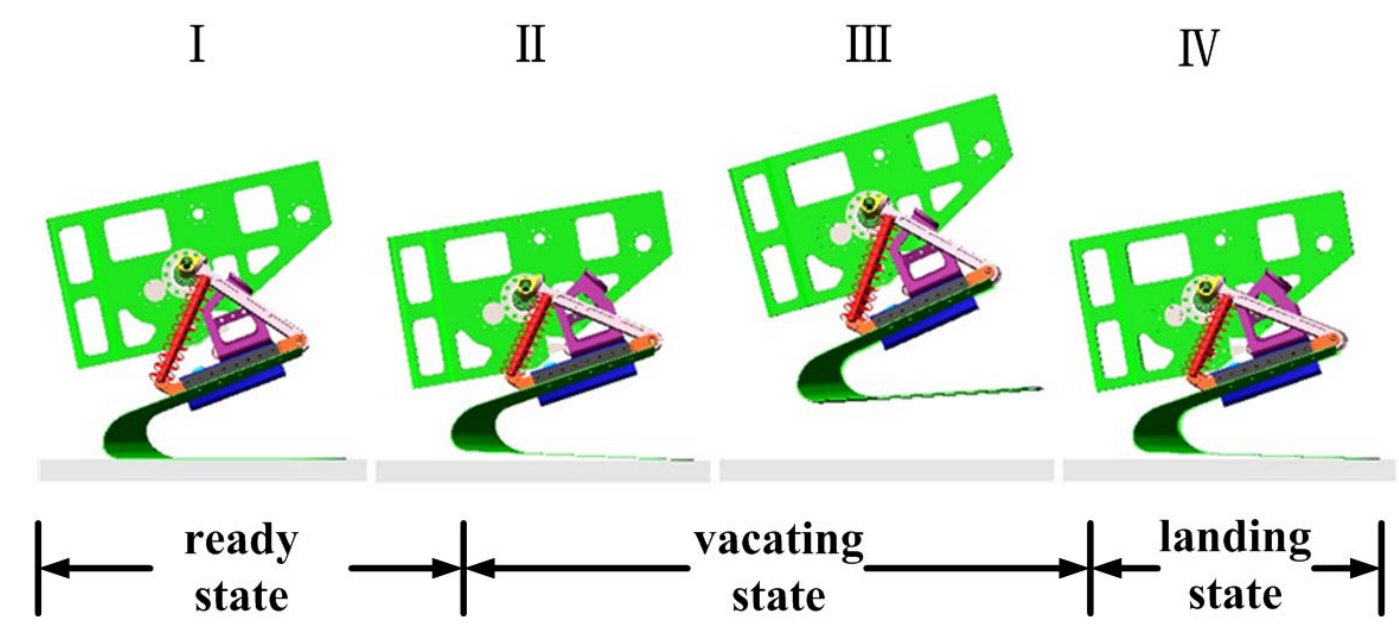

Fig. 7 The different states during the jump simulation

The trajectory of the robot's centroid is shown in Fig. 8. Because the jumping process is in YZ plane, so, the curve $Z$ represents the forward distance of the robot 's centroid position on $Z$ axis, and the curve $Y$ represents the vertical movement locus of the robot's centroid on $Y$ axis, and the curve $\mathrm{X}$ represents the offset of the robot's centroid relative to the $\mathrm{YZ}$ plane. The point a is the beginning of jumping moment, while, the spring has already completed the storage of elastic potential energy. The point $\mathrm{b}$ is the highest point of a single jumping that the robot can get. The point $\mathrm{c}$ is the robot's landing moment, at this time the cam mechanism has already finished a fully compression and the spring has was full of elastic potential energy. The point $\mathrm{d}$ is the farthest forward distance on $\mathrm{Z}$ axis after three jumps. As can be seen from the picture, the robot's average height of a single jump is about $0.13 \mathrm{~m}$ to $0.15 \mathrm{~m}$. After three jumps, the forward distance is about $0.14 \mathrm{~m}$. During the jumping process, the centroid relative to the $\mathrm{YZ}$ plane has a small offset because the curve $\mathrm{X}$ is almost a horizontal line, so it can be judged that the jumping process is relatively stable. In the meantime, because of the curve $\mathrm{Y}$ which is describing the vertical direction of jumping trajectory, showing a smooth and continuous wave type curve, and the curve $Z$ that described the forward direction of jumping trajectory, which is showing a steady upward curve, so it is enough to prove that the whole body is maintaining in a stable and continuous state during the jumping process on YZ plane. The simulation results show that the mechanism design of bionic frog-hopping robot is reasonable.

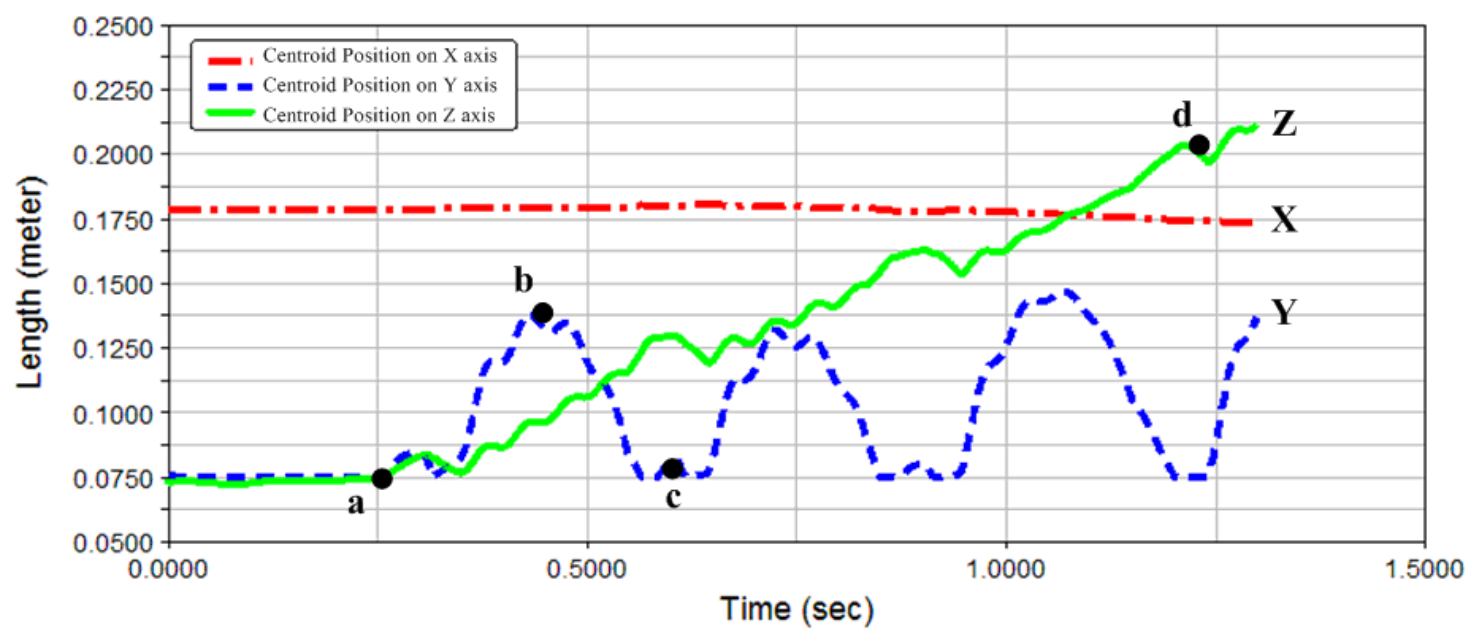

Fig.8 The trajectory of centroid 


\section{Summary}

Comparing to the traditional robot, the bionic frog-hopping robot which can jump continuously and stably shows a better obstacle capability and environmental adaptability. The new type jumping leg improves the storage capacity of elastic potential energy. The addition of C-shape spring sheet makes the jumping leg more flexible and prolongs the service life. The cam mechanism of the bionic frog-hopping robot is the key to achieve continuously jumping. It is simple and novel, and efficient more than traditional structure. Driven by cam mechanism, the two jumping legs can well complete the compression and release in synchronously, which ensures the rollover phenomenon will never happened during the robot's jumping process. The simulation is carried out after the 3D modeling, and simulation results show that the jumping process is as stable as expected. So, this novel structure is feasible, and it will also provides a certain theoretical basis for the further researching on hopping robot.

\section{Acknowledgments}

This project was supported by Scientific Research Common Program of Beijing Municipal Commission of Education under Grant no.KM201410009001, the National Natural Science Foundation of China Grant nos. 51305005, 51375016 and 51402005, Beijing Natural Science Foundation under Grant no. 3122013,

\section{References}

[1] Chen Diansheng, Huang Yu, Wang Tianmiao. Obstacle climbing analysis and simulation of wheel-legged robot. Journal of Beijing University of Aeronautics and Astronautics. 2009,35(3):371-375 (in Chinese)

[2] Graichen K, Hentzelt S, Hildebrandt A, et al. Control design for a bionic kangaroo[J]. Control Engineering Practice, 2015, 42:106-117.

[3] S. Hyon, S. Kamijo, T. Mita. A biologically inspired hopping robot-Kenken. Trans on RSJ, 2002, 20(4):453-462

[4] S. Hyon, T. Emura, T. Mita. Dynamics-based control of one-legged hopping robot. Journal of Systems and Control Engineering, Proceedings of the Institution of Mechanical Engineers Part I, 2003:83-98

[5] S. H. Park. Dynamic modelling and link mechanism of mobile robot.Twenty-Fifth Southeastern Symposium on System Theory, 1993:368-372

[6] Wang Meng. Research on frog-inspired biomimetic jumping robot. Harbin: Harbin Institute of Technology Doctoral Dissertation. 2009. (in Chinese)

[7] S. Hyon, S. Abe, T. Emura. Development of a biologically-inspired biped robotKenken II. Proceedings of 6 th Japan-France Congress on Mechatronics and $4^{\text {th }}$ Asia-Europe Congress on Mechatronics, 2003:404-409

[8] S. Hyon, T. Mita. Development of a biologically inspired hopping robot-Kenken. IEEE International Conference on Robotics and Automation, Washington DC, USA, 2002:3984-3991 